\title{
RDUS
}

Revue de DROIT

UNIVERSITÉ DE SHERBROOKE

$\begin{array}{ll}\text { Titre : } & \text { QUELLE PLACE POUR LE DROIT NATIONAL DANS } \\ & \text { L'ENSEIGNEMENT DU DROIT EN EUROPE? }\end{array}$

Auteur(s) :

Pascal ANCEL

Revue :

RDUS, 2013, volume 43, numéro 1-2

Pages :

89-121

ISSN :

0317-9656

Éditeur: $\quad$ Université de Sherbrooke. Faculté de droit.

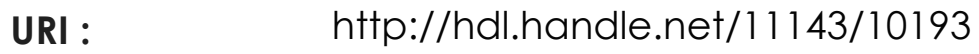

DOI : $\quad$ https://doi.org/10.17118/11143/10193 
Page vide laissée intentionnellement. 


\title{
ARTICLE
}

\section{QUELLE PLACE POUR LE DROIT NATIONAL DANS L'ENSEIGNEMENT DU DROIT EN EUROPE?}

\author{
par Pascal ANCEL*
}

À l'heure d'une mondialisation accélérée, faut-il encore centrer la formation des étudiants juristes sur le droit national, sauf à en spécialiser ensuite certains sur le droit international et le droit comparé? Ne faut-il pas, en renversant la perspective, former d'abord les étudiants à être des juristes du monde, sauf à les spécialiser ensuite dans tel ou tel droit national? À partir de son observation du programme transsystémique de l'Université McGill et de son expérience actuelle à l'Université du Luxembourg, l'auteur examine cette question dans le contexte européen. Si, de son point de vue, il est exclu d'envisager aujourd'hui à grande échelle une transnationalisation de l'enseignement du droit en Europe, tant à raison des besoins encore limités que des obstacles importants au développement de ce type de formation, l'auteur plaide pour la mise en place, dans des contextes limités, d'expériences de transnationalisation dont il examine les conditions et les modalités.

In the face of increasing globalization, is the current focus on legal education of a national nature, with a secondary specialization in international or comparative law, still appropriate? Rather, should one invert the educational process by first giving students a transnational legal education and then providing them exposure to the various legal systems around the world, before offering them the opportunity to specialize in a particular national law? After observing the transsytemic program offered by McGill University, and building upon his current experience at the University of Luxembourg, the writer examines these questions from a European perspective. If, as he concludes, it is simply not possible to implement a largescale transnationalization of the existing legal education system in Europe, not only because of a general lack of need for so called "international lawyers", but also due to the many structural obstacles thereto then, the writer submits, it should nonetheless be possible, within specific contexts, to provide an exposure to the phenomenon of transnationalization.

\footnotetext{
* . Professeur à l’Université du Luxembourg.
} 


\section{SOMMAIRE}

I. Une transnationalisation impossible à l'échelle européenne

A. Des besoins encore limités ..................................... 98

B. Des obstacles actuellement insurmontables ................ 102

1. Obstacles pédagogiques .................................. 102

2. Obstacles culturels ...................................... 107

II. Des expérimentations légitimes dans des contextes particuliers

A. Les conditions d'une expérimentation ....................... 112

B. Les modalités d'une expérimentation ....................... 117 
(2013) 43 R.D.U.S.

Quelle place

pour le droit national

dans l'enseignement du droit en Europe?

Dans le cadre d'un colloque consacré au droit comparé, où on s'interroge notamment sur le rôle et la place de cette discipline dans l'enseignement et la recherche, le titre de cette contribution peut apparaître provocateur, et il a été évidemment choisi pour cela. S'agissant de l'enseignement du droit, il y a une longue tradition consistant à se demander quelle place le droit comparé doit y tenir et comment, selon quelles méthodes, il doit être enseigné. En France, cette problématique avait déjà été abordée par Adhemar Esmein lors du célèbre Congrès international de droit comparé de $1900^{1}$, et elle est, à la même époque, reprise par plusieurs articles de Raymond Saleilles ${ }^{2}$. Après une période de sommeil, l'ampleur du phénomène de mondialisation - ou de globalisation -, en même temps que le développement des processus d'harmonisation des droits nationaux en Europe, l'a fait ressurgir avec vigueur dans la littérature juridique contemporaine ${ }^{3}$. Toutes ces réflexions - dont il y a naturellement beaucoup à tirer, et qui se sont prolongées dans le cadre du congrès de Sherbrooke - partent cependant du présupposé selon lequel l'enseignement du droit est (et, pour beaucoup, doit être) d'abord l'enseignement du droit national, à côté duquel on cherche à introduire, au titre d'une ouverture vers le monde, une formation plus ou moins approfondie, et plus ou moins intégrée aux diverses matières, des systèmes juridiques étrangers. Telle est effectivement la situation dans l'immense majorité des facultés de droit (ou des écoles de droit), au Québec et en Europe, même si le droit national qu'on y enseigne à titre principal est aujourd'hui largement mâtiné de concepts venus d'ailleurs, et complété, à l'intérieur même de l'ordre juridique interne, par des règles supranationales ou transnationales invocables, parfois avec primauté de rang, devant les juridictions du

1. Adhémar Esmein, "Le droit comparé et l'enseignement du droit ", Congrès international de droit comparé, 1900.

2. Raymond SALEILlEs, "Les méthodes d'enseignement du droit et l'éducation intellectuelle de la jeunesse ", Revue internationale de l'enseignement, (1902) t. 44, 313-329; "L'enseignement du droit. Lettre de M. R. Saleilles à M. P. Desjardins ", Revue internationale de l'enseignement, (1908) t. 56, 289-310; "Droit civil et droit comparé ", Revue internationale de l'enseignement, (1911) t. 61, 5-32.

3. Voir notamment Bénédicte FAUVARQUE-COSSON, "L'enseignement du droit comparé ", Revue internationale de droit comparé, (2002) n² 2, 293-309. 
pays ${ }^{4}$. Mais, à l'heure d'une mondialisation accélérée, on peut se demander s'il ne convient pas de commencer à dépasser cette approche, et à remettre en cause le présupposé : faut-il, dans notre village planétaire, continuer à centrer la formation des étudiants juristes sur le droit national, sauf à en spécialiser ensuite certains sur le droit international et le droit comparé? Ne faudrait-il pas, en renversant la perspective, les former d'abord à être des "juristes du monde ", des " global lawyers ", pour ensuite en faire (éventuellement) des spécialistes de droit français, québécois, luxembourgeois...?5

Si cette question mérite d'être posée au Québec, c'est parce qu'un tel renversement de perspective y a été expérimenté depuis quelques années, avec un certain succès, dans le cadre de l'Université McGill, avec la mise en place, à la fin des années 1990, du programme transsystémique. La Faculté de droit de McGill, qui a une longue tradition comparatiste, cherche depuis longtemps à répondre au défi de la formation des étudiants en droit dans un pays marqué par le bijuridisme, où les juristes sont inévitablement confrontés, au cours de leur vie professionnelle, aux deux systèmes du common law et du droit civil en vigueur au Québec. Dans ce but, cette faculté avait d'abord imaginé, dès 1968, un cursus dit "national " dans lequel les étudiants suivaient successivement une formation dans l'un et l'autre système, en commençant par celui de leur choix ${ }^{6}$. Cependant, l'expérience avait montré que, dans cette formule, la pensée juridique des étu-

4. Je songe naturellement aux règles du droit européen - qu'il s'agisse des règles de l'Union européenne ou de celles tirées de la Convention européenne des droits de l'homme - règles qui, en France comme dans tous les pays d'Europe, ont dû être intégrées, bon gré mal gré, dans les enseignements où elles sont mises sur le même plan que les règles nationales dont elles commandent du reste l'évolution.

5. Sur la problématique de l'enseignement du droit à l'ère de la globalisation voir en général : Harry ARTHURS, "Law and Learning in an Era of Globalization", (2009) 10 German Law Journal 639.

6. Sur ce programme national, voir RODERICK A. MACDONALD, "The National Law Programme at McGill: Origins, Establishments, Prospects ", (1990) 13 Dalhousie L.J. 211. Adde : John E.C. BRIERLEY, "Developments in Legal Education at McGill, 1970-1980", (1982) 7 Dalhousie L.J. 364. 
(2013) 43 R.D.U.S.

Quelle place

pour le droit national

dans l'enseignement du droit en Europe?

diants, leur manière de raisonner, d'argumenter, étaient irrémédiablement marquées, formatées, par le système auquel ils avaient été formés en premier lieu 7 . D'où l'idée d'aller plus loin et de créer un programme d'enseignement qui ne se situe pas, au départ, à l'intérieur d'un système juridique national, qui forme les étudiants, de manière intégrée, en même temps au common law et au droit civil québécois, et même, au-delà, au moins chez certains promoteurs du programme, à être des juristes aptes à appréhender n'importe quel système juridique, étatique, supra-, infra- ou trans-étatique 8 . J'ai eu la chance de pouvoir observer cette expérience in vivo parce que, dans le cadre d'un récent séjour d'études au Québec, j'ai pu me transformer en étudiant et suivre la plus grande partie du cours de droit des contrats de Jean-Guy Belley ainsi que, de manière plus épisodique, les enseignements d'autres collègues $^{9}$. Même si je sais que ce programme est contesté ici même, au Québec, et même si je suis bien conscient qu'il est lié à un contexte tout à fait particulier, je dois dire que j'ai été quelque peu fasciné, après d'autres, par cette tentative de "dénationaliser " l'enseignement du droit, qui constitue une véritable révolution dans la manière d'appréhender la formation des juristes ${ }^{10}$. Que les objectifs ambitieux de ce programme aient été atteints ou non est

7. Helge Dedek et Armand De Mestral, "Born to be Wild: The Transsystemic Program at McGill and the De-Nationalization of Legal Education ", (2009) German Law Journal, vol. 10, 889 s., spéc. 903-904 : "le programme juxtaposait (les deux traditions juridiques) de telle sorte que les étudiants à la faculté de droit étaient marqués comme appartenant à l'une ou l'autre des deux traditions... Au fil du temps, il était devenu apparent que les étudiants qui avaient été exposés à un an de formation juridique n'apprendraient pas les bases de l'autre tradition de la même manière que ceux qui l'avaient choisie en première année".

8. Pour une telle approche : Roderick A. MACDONALD et Jason MACLEAN, " No Toilets in Park ", (2005) 50 McGill Law Journal 721.

9. Pour une réflexion plus complète à partir de cette expérience, je me permets de renvoyer à mon article: Pascal ANCEL "Dénationaliser l'enseignement du droit civil? Réflexions à partir d'une expérience québécoise ", (2011) RTD civ. 701-724.

10. Un professeur américain a pu comparer le programme transsystémique à l'introduction de la méthode socratique par Langdell à la fin du XIXe siècle : Peter L. STRAuss, "Transsystemia - Are We Approaching a New Langdellian Moment? Is McGill Leading the Way? ", (2006) 24 Penn State International Law Review 763. 
une question dans laquelle je me garderai bien de rentrer ici, et sur laquelle je n'ai du reste pas suffisamment d'éléments de réponse; l'aventure transsystémique, qui dure depuis plus de 10 ans, montre au moins qu'il est possible de concevoir une formation juridique qui ne soit pas centrée, au départ, sur la seule acquisition des savoirs locaux. Elle amène donc très directement à s'interroger sur la place que doit tenir aujourd'hui le droit national dans la formation des juristes, en dépassant la question classique de l'enseignement du droit comparé.

Cette manière nouvelle de poser la question devrait aujourd'hui tout particulièrement interpeller le monde universitaire européen. Au-delà de l'instauration progressive d'un nouvel ordre juridique qui, à l'intérieur de chaque pays membre de l'Union européenne, se superpose aux ordres nationaux, l'Europe d'aujourd'hui se caractérise par un développement sans précédent des activités et des relations transfrontalières, ainsi que par une circulation accrue des juristes (praticiens et universitaires), mais aussi (ceci expliquant cela) des idées et des concepts juridiques d'un pays à l'autre. Dans ce contexte, l'idée que les facultés de droit, en Europe, devraient d'abord former des juristes européens (plutôt que des juristes français ou allemands ou espagnols) peut exercer un certain pouvoir de séduction. Dès avant la mise en place de l'expérience de McGill, un certain nombre d'auteurs avaient déjà prôné une certaine dénationalisation de l'enseignement du droit dans le cadre européen. Ainsi, dans un article paru en Allemagne en 1996, Axel Flessner, reprenant des idées déjà exprimées par Heinz $\mathrm{Kötz}^{11}$, se prononçait en faveur d'une "formation des juristes qui traite en premier des champs principaux du droit en Europe comme un système unifié dans lequel les systèmes juridiques nationaux sonnent comme des varia-

11. Heinz KöTz, "A Common Private Law for Europe: Perspectives for the Reform of European Legal Education " dans Bruno de WITTE \& Caroline FORDERS (dir.), The Common Law in Europe and the Future of Legal Education, Kluwer, 1992, 31-38; du même auteur : " Europäische Juristenausbildung", Zeitschrift für europäisches Privatrecht, 1993, 268-272. 
(2013) 43 R.D.U.S.

Quelle place

pour le droit national

dans l'enseignement du droit en Europe?

tions de thèmes généraux plus larges "12. Et depuis 1999, plusieurs auteurs (à vrai dire issus eux-mêmes de McGill) se sont interrogés sur la possibilité et l'opportunité de transposer le programme transsystémique dans le contexte européen ${ }^{13}$. Cependant, jusqu'à maintenant, très rares sont, en Europe, les expériences de formation juridique transnationale menées au stade des premières années. Certes, beaucoup d'universités ont mis en place des masters spécialisés en droit transnational (ou global), mais il s'agit de formations supérieures destinées à des étudiants avancés qui ont été, au départ, formés à leur droit national14. Plusieurs grandes universités européennes ont par ailleurs passé des accords permettant à leurs étudiants de suivre une formation binationale, par alternance dans l'une puis dans une autre université15. Mais quel que soit l'intérêt tout à fait considérable de ces cursus, ils restent

12. Axel Flessner, "Deutsche Juristenbildung : Die kleine Reform und die europäische Perspektive ", 51 Juristenzeitung, (JZ) 689, 691.

13. On consultera en particulier: Armand de MESTRAL, "Bisystemic Law Teaching - The McGill Programme and The Concept of Law in the EU ", (2003) Common Market Law Review, vol. 40, 799-807; Helge DEDEK et Armand de Mestral, "Born to be Wild: the Transsystemic Program at McGill and the De-Nationalization of Legal Education", (2009) German law journal, vol. 10, 889 s.; Pierre LAROUCHE, "L'intégration, les systèmes juridiques et la formation juridique ", (2000-2001) 46 McGill Law Journal 1011.

14. On notera de même avec intérêt que certaines formations professionnelles aux " métiers du droit " intègrent désormais une dimension internationale, alors même que la profession à laquelle elles préparent doit s'exercer dans un cadre principalement national. Il en va ainsi du programme de formation initiale de l'École nationale de la magistrature (sur lequel on consultera le site Web de l'École: http://www.enm.jus tice.fr/_uses/lib/5762/Programme_pedagogique_2011.pdf). Mais il s'agit là encore d'une internationalisation a posteriori, qui s'adresse à des juristes déjà formés à l'université au droit national français).

15. Sur ces programmes, voir Anne KLEBES-PELISSIER, "Double Degrees in the Context of the Bologna Process ", 4 European Journal of Legal Education (Eur. J. Leg. Educ.), (2007) 173; Audrey Guinchard, "The Double Degree Experience between England and France: A Contribution to an Integrated European Legal Education", (2007) 4 Eur. J. Leg. Educ., 3. Voir, par exemple, le programme du partenariat entre Cologne et Paris I, consultable sur: http://www.mastercologneparis.info/, ou, en dehors de la France, le programme "hanséatique" entre Groningen, Bremen, et Oldenburg (http://www.hanse-law-school.de/about_hls.htm). 
dans une logique de juxtaposition de formations axées sur un système national. Même si on peut trouver quelques éléments de transnationalisation dans le programme de l'École de droit de Sciences Po Paris créée en 200916, le seul cursus de formation initiale véritablement détaché d'un droit national était jusque-là, à ma connaissance, le bachelor de l'European Law School de Maastricht, mis en place en 2006, qui offre, dès la première année, à des étudiants titulaires d'un diplôme de fin d'études secondaires (le baccalauréat français) une formation au droit des contrats, au droit de la responsabilité, au droit pénal... sans rattachement national ${ }^{17}$. Cependant, l'idée commence à faire son chemin ${ }^{18}$, et elle nourrit le projet de refonte du programme de bachelor qui est actuellement en cours d'élaboration au sein du Département de droit de 1'Université du Luxembourg. Ce projet19, qui était déjà en discussion avant mon arrivée dans cette université, mais à la préparation duquel je suis maintenant directement associé, sera donc évidemment au cœur des réflexions que j'entends mener ici sur l'intérêt et la possibilité de mettre en place en Europe des programmes de formation transnationalisés plus ou moins inspirés de l'expérience de McGill.

Il est clair que, quelle que soit la fascination que peut exercer cette expérience, il faut faire preuve d'une grande prudence lorsqu'on réfléchit à la possibilité de la transposer dans le contexte européen. De mon point de vue, et ce sera le premier temps de ma

16. Sur laquelle on consultera Christophe JAMIN, La cuisine du droit, L'école de Droit de Sciences Po : une expérimentation française, Lextenso éditions, 2012.

17. Sur le programme de Maastricht, voir: Aalt Willem HeRINGA et Bram AKKERMANS (dir.), Educating European Lawyers, Intersentia, Metro, 2011.

18. Un second programme transnational, plus radical encore, et fortement teinté de pluridisciplinarité, vient d'être mis en place à l'Université de Tilburg, sous la direction de Pierre LAROUCHE, issu lui-même de McGill (Bachelor in Global Law, sur lequel on consultera le site Web de l'Université de Tilburg: http://www.tilburguniversity.edu/education/ bachelors-programmes/globallaw/.

19. Je tiens à préciser qu'il ne s'agit pour l'instant que d'un projet interne au Département de droit, dont les modalités de mise en place sont actuellement en cours de discussion au sein des instances universitaires. L'objectif serait d'ouvrir ce nouveau bachelor à la rentrée 2014. 
(2013) 43 R.D.U.S.

Quelle place

pour le droit national

dans l'enseignement du droit en Europe?

réflexion, il est aujourd'hui totalement irréaliste, et sans intérêt, d'imaginer une généralisation en Europe de formations juridiques transnationalisées, qui feraient passer au second plan la formation aux systèmes juridiques nationaux des différents États, de toute façon indispensable. Il n'est pas difficile de le montrer, tant les multiples arguments qu'on peut invoquer en ce sens correspondent à une conception de l'enseignement du droit profondément ancrée dans l'esprit de l'immense majorité des universitaires européens. Quitte à avoir l'air d'enfoncer des portes ouvertes, il vaut cependant la peine d'examiner dans le détail ces multiples arguments. Outre que certains d'entre eux doivent être dès maintenant relativisés, il faut en effet avoir conscience que, dans un contexte international en pleine évolution, ils sont susceptibles de perdre assez vite de leur pertinence. Aussi bien - et c'est ce que je voudrais développer dans un second temps - il y a actuellement place en Europe, dans certains contextes particuliers, pour des expérimentations qui pourraient ouvrir la voie à des évolutions futures à un niveau plus général. L'impossibilité actuelle d'une généralisation de formations transnationales en Europe (I) ne doit surtout pas conduire à remettre en cause la légitimité des expérimentations (II).

\section{Une transnationalisation impossible à l'échelle euro- péenne}

Lorsqu'on évoque actuellement, dans des discussions avec des collègues européens, la possibilité de transposer en Europe un programme du type de celui de McGill, on s'entend généralement répondre que cela ne correspond ni aux besoins des étudiants que nous formons, ni aux attentes des praticiens qui les embaucheront, et, surtout, que la mise en place d'un tel programme se heurterait à des obstacles insurmontables dans la plupart des universités européennes. Ces arguments sont imparables, mais ils doivent être relativisés. S'ils sont actuellement limités, les besoins de formations transnationales peuvent très vite évoluer (A). Quant aux obstacles, ils ne sont aujourd'hui insurmontables que parce que la culture universitaire dominante ne peut guère envisager de les faire tomber (B). 
Quelle place

pour le droit national

(2013) 43 R.D.U.S.

dans l'enseignement du droit en Europe?

\section{A. Des besoins encore limités}

De prime abord, une formation qui, dès le départ, n'est pas axée sur le droit national du pays où se déroule l'enseignement ne paraît apte à former qu'un nombre limité de juristes qui, dans le cadre de leur activité professionnelle future, seront spécialement confrontés à des rapports juridiques internationaux. En ce sens, le programme transsystémique de McGill paraît surtout avoir été conçu pour former les futurs collaborateurs de grands cabinets d'avocats internationaux, qui du reste, d'après les informations qu'on peut avoir, semblent particulièrement friands des étudiants ainsi formés. Dans son récent et passionnant ouvrage où il relate l'expérimentation de l'École de droit de Sciences Po Paris, Christophe Jamin note à juste titre la grande diversité des profils que la pratique attend des juristes formés à l'université20. À cet égard, il est clair que les compétences attendues d'un futur notaire devant travailler dans un milieu rural n'ont rien à voir avec celles que devrait avoir un avocat ou un juriste d'entreprise spécialisé dans la rédaction de contrats ou dans le règlement de litiges internationaux. Or, dans l'Europe d'aujourd'hui, il paraît évident que l'immense majorité des juristes formés par les facultés de droit sont encore des juristes qui auront une activité essentiellement locale, de sorte qu'il serait absurde de concevoir, pour tous, des programmes de formation détachés du système juridique national. C'est ce que souligne à juste titre un professeur français en observant que

parmi l'ensemble des professionnels du droit recrutés chaque année, bien peu font du droit international... et, finalement, assez peu ont l'impérieuse nécessité de parler plusieurs langues. La vie quotidienne d'un magistrat, d'un avocat, d'un notaire français se déroule en français dans un cadre de droit français (... et communautaire et européen, mais en français) et ces activités nécessitent avant tout une connaissance technique des règles de droit positif, une capacité de raisonnement logique, une aptitude à

20. C. JAMIN, préc., note 16, 129-140. 
(2013) 43 R.D.U.S.

Quelle place

pour le droit national

99

dans l'enseignement du droit en Europe?

l'expression écrite et orale claire, bref tout ce à quoi les facultés de droit savent former depuis leur origine ${ }^{21}$.

S'il est difficile de contester cette opinion, il faut cependant observer que tous les juristes européens, même ceux qui ont de prime abord une activité purement locale, sont aujourd'hui concernés par le phénomène de globalisation et d'internationalisation des relations juridiques. Je ne parle pas bien entendu ici du phénomène d'européanisation du droit auquel tous nos étudiants seront, dans leur vie professionnelle, inévitablement confrontés, dans la mesure où les règles d'origine européenne sont incorporées dans les ordres juridiques nationaux des pays membres de l'Union européenne, et où de toute façon, même une formation juridique strictement nationale doit (ou devrait) les intégrer ${ }^{22}$. Il n'y a là rien qui relève à proprement parler d'une transnationalisation, même si les progrès constants de l'harmonisation européenne sont de nature à favoriser une circulation des concepts et des modes de raisonnement entre les différents systèmes juridiques. Et sans doute peut-on penser qu'un juriste formé d'une manière non exclusivement nationale serait plus apte à appréhender un tel phénomène que celui à qui on a présenté le système national comme une vérité, faisant apparaître comme anormal tout ce qui vient d'ailleurs. Mais, abstraction faite de cette européanisation des droits nationaux, il faut avoir conscience que la facilité de circulation des personnes et des biens, le développement constant des échanges économiques transfrontaliers à l'intérieur et même au-delà de l'espace européen, aboutissent à multiplier les problèmes dont la solution ne suppose pas seulement 1'habile maniement du droit national. Quel notaire français,

21. Hervé CROZE, "La diversité des Écoles de droit - À propos de l'école de droit de l'institut d'études politiques de Paris ", (2009) JCP G, n 44, 376.

22. Je dis "doit ou devrait ", car je ne suis pas sûr que ces règles reçoivent toujours la place qu'elles méritent dans la formation des étudiants des différents pays d'Europe (et en tout cas en France), en dehors des enseignements de "droit communautaire " qui leur sont spécialement consacrés. Souvent vécues comme un facteur de perturbation, de désordre, des systèmes juridiques nationaux, elles sont parfois marginalisées dans les enseignements des différentes matières qu'elles affectent (spécialement dans l'enseignement du droit civil). 
même travaillant à la campagne, n'a jamais eu l'occasion de rédiger une vente immobilière à des étrangers, de régler une succession présentant des éléments internationaux ou un partage de biens entre des époux de nationalité différente? Qui peut dire ce qu'il en sera dans 20 ans, et quelle place le règlement de questions internationales occupera dans l'activité des juristes que nous formons aujourd'hui? Cela bien entendu ne suffit pas à justifier une transnationalisation à grande échelle de nos programmes de formation actuels, mais cela doit faire prendre conscience que le modèle d'une formation centrée sur le droit national n'est peut-être pas un modèle éternel et que sa pertinence par rapport aux besoins de nos étudiants doit, à tout le moins, être périodiquement réinterrogée.

On pourrait du reste, même dans le contexte actuel encore largement marqué par la suprématie des droits nationaux, être tenté de nuancer l'affirmation liminaire selon laquelle une formation transnationalisée ne serait apte qu'à former des "global lawyers ", donc pour l'instant une infime minorité de juristes. Contrairement à l'enseignement classique, qui (au moins dans les pays de civil law) est axé sur la présentation systématique aux étudiants des solutions du droit positif, une formation transnationale oblige l'enseignant à construire son enseignement à partir des problèmes, des questions auxquels les différents droits nationaux donnent des réponses ${ }^{23}$. Certes, cette entrée à partir des problèmes semble familière aux juristes de common law, elle paraît être consubstantielle à la méthode socratique. Mais, dans une formation transnationalisée, les problèmes qui servent de grille à la construction des cours ne sont pas des problèmes internes à un système national, ce sont des problèmes communs à plusieurs systèmes, et la recherche des solutions actuelles ou prévisibles dans tel ou tel droit n'est pas le but premier de l'enseignement. Les solutions des différents droits nationaux ne sont que des exemples permettant de comprendre la problématique, de mesurer les enjeux des choix entre les différentes manières d'y répondre.

23. Voir là-dessus les exemples donnés dans P. ANCEL, préc., note 9, spec. nos $12-15$. 
(2013) 43 R.D.U.S.

Quelle place

pour le droit national

dans l'enseignement du droit en Europe?

Or, il n'est pas exclu qu'une telle manière de former les étudiants, au-delà de l'objectif premier consistant à former des juristes aptes à se mouvoir au sein de différents systèmes nationaux, ne leur permette pas aussi de mieux appréhender le système au sein duquel ils seront principalement amenés à évoluer au cours de leur activité professionnelle. Que l'on réfléchisse un instant aux défauts de la formation dogmatique que nous dispensons actuellement, et qui prétend donner aux étudiants la vision la plus complète possible des solutions du droit positif. Cette manière traditionnelle d'enseigner se heurte aujourd'hui au caractère de plus en plus complexe et à l'évolution de plus en plus rapide des solutions, même au sein des systèmes juridiques nationaux. Pour prétendre bien former les étudiants dans un tel contexte, la tentation est grande de multiplier les cours et les heures d'enseignement. Mais on se lance alors dans une course illusoire et perdue d'avance. L'expérience qu'on peut avoir des examens écrits ou oraux montre que les étudiants ont de plus en plus de mal à hiérarchiser au sein de l'immense masse de connaissances dont ils sont gavés tout au long de leurs études, à distinguer entre les principes de base, dotés d'une certaine permanence, et les solutions de détail susceptibles d'une rapide obsolescence. Elle montre aussi que les étudiants sont mal préparés à résoudre de manière imaginative les multiples problèmes dont la solution ne leur a pas été préalablement exposée par leur professeur, et auxquels pourtant, dans leur vie professionnelle, ils seront inévitablement confrontés. À tout le moins, l'observation de la manière dont peut fonctionner un programme transnationalisé devrait nous inciter, même dans le cadre des formations classiques axées sur le droit national, à centrer nos enseignements plus sur les problèmes que sur les réponses d'un droit positif marqué par une instabilité croissante. Cela, cependant, ne conduit pas nécessairement à renoncer à la focalisation de nos enseignements sur le droit national, surtout si on considère les obstacles auxquels se heurterait aujourd'hui, dans la plupart des universités européennes, une formation juridique transnationalisée. 


\section{B. Des obstacles actuellement insurmontables}

Les obstacles qui viennent à l'esprit sont d'abord d'ordre pédagogique, tenant tant aux difficultés que les étudiants, dans la plupart des universités européennes, auraient à suivre une telle formation qu'à la difficulté de recruter des équipes de professeurs ayant les compétences pour l'assurer. Cependant, si ces difficultés tenant aux systèmes universitaires en place dans la plupart des pays sont incontestables, elles sont sans doute moins fondamentales que les obstacles d'ordre culturel liés aux systèmes juridiques constituant actuellement la base des enseignements.

\section{Obstacles pédagogiques}

D'un point de vue pédagogique, on mettra le plus souvent en avant les difficultés qu'un enseignement détaché des droits nationaux présenterait pour les étudiants, et les risques qu'il leur ferait encourir. Dans l'esprit des promoteurs de l'enseignement transsystémique à McGill, il s'agissait, à l'origine du moins, de former des juristes qui, au lieu d'être marqués, formatés $a b$ initio, en tant que juristes de droit civil ou de common law, seraient aussi à l'aise dans les deux systèmes, parleraient en quelque sorte aussi parfaitement les deux droits - comme un enfant qui, dans un couple mixte, fait en même temps l'apprentissage des deux langues de ses parents ${ }^{24}$. Cependant, la comparaison a ses limites et, à la différence de l'acquisition du langage, qui se fait en quelque sorte de manière naturelle, l'apprentissage de deux systèmes juridiques en même temps semble requérir de la part des étudiants une capacité d'attention et un travail considérables, beaucoup plus importants que ceux qui sont nécessaires pour la formation à un seul droit national. D'une part, le fait d'enseigner plusieurs systèmes juridiques en même temps conduit nécessairement à consacrer moins de temps à chacun d'entre eux, à faire des " coupes sombres" dans les matières enseignées, de sorte que l'étudiant sera obligé de se documenter par lui-même pour avoir

24. Pour cette comparaison, voir en particulier: Daniel JUTRAS, "Énoncer l'indicible : le droit entre langues et traditions ", (2000) 52 RIDC 781-786. 
(2013) 43 R.D.U.S.

Quelle place

de chaque système une connaissance suffisante. D'autre part, on peut craindre que le passage continuel d'un système à l'autre ne conduise les étudiants à faire des mélanges entre les règles, les solutions ou les méthodes de raisonnement. Le risque, souvent évoqué ici même au Québec, est alors que les juristes ainsi formés ne maîtrisent correctement aucun des systèmes ou, pire, qu'ils les mélangent et ne parlent qu'une sorte de sabir qui ne serait compréhensible ni devant un tribunal d'un pays civiliste, ni devant une juridiction de common law. Ce risque paraît assez bien maîtrisé à McGill, comme l'attestent les résultats obtenus : non seulement les étudiants qui sortent de cette université semblent particulièrement prisés des grands cabinets d'affaires internationaux, mais, contrairement à ce que beaucoup de collègues québécois semblaient penser, ils connaissent une réussite remarquable aux examens professionnels de l'École du Barreau du Québec, pourtant fortement axé sur le droit national de la province ${ }^{25}$. Ces résultats peuvent cependant s'expliquer par le contexte particulier de 1'Université McGill, qui recrute des étudiants triés sur le volet, ayant déjà suivi, pour la plupart, une formation universitaire généraliste selon le modèle des États-Unis. Ces étudiants, par ailleurs parfaitement bilingues, sont censés avoir une capacité de travail et de réflexion suffisante pour jongler constamment entre les deux systèmes, sans les mélanger. Or, ces conditions ne se retrouvent pas dans l'immense majorité des universités européennes, l'exemple français étant à cet égard particulièrement significatif.

Dans le système universitaire français, les étudiants - qui commencent en principe les études de droit à 18 ans immédiatement après le baccalauréat - ne sont normalement pas sélectionnés à l'entrée dans l'enseignement supérieur: la réussite au

25. Pendant l'année de mon séjour au Québec, en 2010, McGill avait obtenu les meilleurs résultats de toute la province à ces examens. Elle avait, à la surprise de beaucoup, détrôné l'Université de Sherbrooke, axée sur une formation plus directement professionnalisante, et qui occupait traditionnellement la meilleure place. 
baccalauréat donne le droit d'accès à l'université26. Il en résulte, dans les premières années de la formation juridique dans les facultés de droit, des auditoires à la fois pléthoriques et très hétérogènes, devant lesquels, de l'avis général, il paraîtrait peu réaliste de conduire des enseignements transnationalisés. Seule une petite minorité d'étudiants semble disposer de suffisamment d'autonomie pour compléter par l'étude des manuels les connaissances forcément rudimentaires en droit national qui leur seraient présentées par leurs professeurs dans le cadre de tels enseignements. On imagine aisément, alors, que le taux d'échec des étudiants, déjà très élevé avec des enseignements de type classique, deviendrait catastrophique si les étudiants étaient confrontés à des cours naviguant sans cesse d'un système à l'autre, nécessairement plus complexes et plus "théoriques". L'argument paraît d'autant plus fort que, dans le contexte européen, il n'y a pas de raison de centrer la formation des étudiants, comme c'est le cas au Québec, sur une confrontation des deux grands modèles que sont le common law et le civil law. Si on voulait sortir l'enseignement du droit des cadres nationaux, il faudrait tout autant l'ouvrir à la diversité des systèmes civilistes - l'opposition des traditions française et allemande étant au moins aussi prégnante en Europe que celle entre droit civil et common law - et cela rendrait la transposition en Europe d'un enseignement de type " transsystémique " plus difficile encore, et plus inaccessible à nos étudiants ${ }^{27}$.

26. En Europe, les conditions d'accès à l'université varient énormément d'un pays à l'autre. On peut cependant considérer que la majorité des pays d'Europe continentale ne pratiquent aucune sélection à l'entrée dans l'enseignement supérieur (sous réserve de certaines filières où il existe un numerus clausus). Il en va autrement en Grande-Bretagne, où chaque établissement fixe de manière autonome ses conditions d'accès. Pour une description comparative des études de droit dans les différents pays européens, on pourra consulter: Christian BALDUS, Thomas FINKENAUER et Thomas RÜFnER (dir.), Bologna und das Rechtsstudium, Mohr Siebeck, 2011.

27. Comp. A. DE Mestral et H. DedeK, préc., note 7, 910 : " the process of mixing, merging and blending is surely even more complex in the EU than in mixed jurisdictions where only two legal traditions meet ". 
(2013) 43 R.D.U.S.

Quelle place

Ces arguments ne peuvent pas être négligés, mais ils doivent cependant être relativisés. À cet égard, sans prétendre répondre à toutes les objections précédentes, je voudrais faire deux remarques. D'une part, il y a beaucoup d'ambiguïtés sur ce que pourrait être un enseignement transnationalisé : beaucoup de collègues s'imaginent qu'il s'agit d'enseignements de droit comparé où on confronte systématiquement, sur chacune des questions abordées, les solutions des autres droits nationaux ou du moins de ceux qui apparaissent les plus représentatifs. Dans une telle représentation, les cours transnationaux paraissent, indépendamment de la difficulté qu'ils sont supposés représenter pour les étudiants, impossibles à assurer dans les contraintes horaires de l'enseignement universitaire, sauf à ne donner de chaque système qu'une vue extrêmement superficielle. L'objection tombe en partie cependant si on pose que ces cours doivent se focaliser davantage sur la présentation des problèmes que sur une comparaison systématique des solutions, celles-ci n'étant prises que comme des illustrations possibles de différents modèles de régulation, des différentes manières d'appréhender juridiquement telle ou telle question d'organisation sociale. Cela ne change rien, bien entendu, au fait que les étudiants ne se verraient présenter leur droit national que de manière très lacunaire et qu'ils devraient très largement le découvrir par eux-mêmes. Mais tout le pari de ce type de formation est qu'elle donnerait à ceux qui la suivent des clés plus adaptées pour leur permettre cette découverte que celles que procure la formation classique, de toute façon elle-même inévitablement incomplète. D'autre part, il faut peut-être soumettre à un examen critique l'opinion selon laquelle des cours transnationaux seraient nécessairement plus complexes, plus difficiles à suivre par les étudiants moyens que les cours traditionnels fondés sur une étude plus ou moins approfondie du droit national. Il faut avoir conscience que l'assimilation d'une science juridique nationale présente elle-même un degré élevé de complexité, et qu'il n'est peut-être pas a priori plus difficile de faire comprendre à un étudiant quel est le problème et quels sont les grands modèles de solutions possibles à l'aide de quelques exemples bien choisis que de lui faire assimiler tous les raffinements d'une analyse doctrinale ou d'une construction jurisprudentielle ainsi que toutes les dis- 
tinctions conceptuelles internes à un système juridique donné. En France, comme dans d'autres pays, le premier semestre du cours de première année de droit constitutionnel est, depuis longtemps, consacré à la "théorie de l'État ": il ne s'agit pas d'exposer aux étudiants le détail du système constitutionnel français (cela viendra ensuite), mais de leur présenter, d'un point de vue historique et comparatiste, les différents modes d'organisation de l'État (monarchie/république, système parlementaire/présidentiel, etc.). Or, il n'est pas du tout certain que ce cours, bien que plus "théorique ", soit plus difficile d'accès pour les étudiants que celui de droit civil, où on enseigne, par exemple, toutes les subtilités de la distinction, en droit français, des actes juridiques et des faits juridiques, ou des actes de disposition et d'administration... Si nous sommes naturellement portés à penser qu'il serait trop difficile d'enseigner de la même manière aux étudiants, dans le cadre d'une approche transnationale, les grands modèles de solutions en droit de la responsabilité civile ou de droit des contrats, c'est peutêtre parce que nous avons été nous-mêmes formés à une approche nationale et dogmatique de ces matières et qu'il est évidemment plus facile pour nous de reproduire cette forme d'enseignement que de construire un tout autre type de cours. Il n'est pas sûr en revanche qu'un étudiant de première année, qui n'aurait pas connu autre chose, trouve plus difficile d'avoir un cours transnational de droit des contrats ou de la responsabilité civile que de devoir assimiler le système national dans toute sa complexité.

On débouche alors cependant sur une autre objection, sans doute plus importante que les précédentes, et qui tient cette fois non plus aux étudiants, mais au corps académique chargé de les former. L'expérience transsystémique n'a pu être mise en place à McGill que parce que la Faculté de droit y rassemble une équipe de professeurs d'origines nationales très diverses, qui, même s'ils ont nécessairement été formés individuellement à l'intérieur d'un système, sont aptes, à travers les échanges et le travail collectifs, à concevoir et à pratiquer des enseignements qui sortent des cadres nationaux traditionnels. Rien de tel dans l'immense majorité des universités européennes, où le corps enseignant est presque exclusivement composé de juristes eux-mêmes formés dans le moule 
(2013) 43 R.D.U.S.

Quelle place

pour le droit national

dans l'enseignement du droit en Europe?

du droit national. C'est sans doute le cas en France plus qu'ailleurs à raison du caractère national des procédures de recrutement, qui rend l'accession au grade de professeur ou même de maître de conférences très difficile à des juristes, même de très haut niveau, qui n'auraient pas été nourris au lait de la formation universitaire française. Même s'il ne manque évidemment pas, dans les universités françaises, de comparatistes de renom, ceuxci ne couvrent pas l'ensemble des champs disciplinaires, et il paraît extrêmement difficile de constituer des équipes d'enseignants qui auraient les compétences nécessaires pour monter des cours transnationaux dans toutes les matières fondamentales. Le recours épisodique aux conférenciers étrangers et aux professeurs invités ne pourrait constituer qu'un pis-aller, clairement insuffisant pour assurer une cohérence dans les enseignements et un suivi suffisant des étudiants ${ }^{28}$. Il faut ajouter que, au-delà de la question des compétences, on aurait du mal à trouver, dans la plupart des universités françaises, des enseignants suffisamment motivés pour mettre en place de tels enseignements transnationalisés, tant est prégnant le modèle universitaire traditionnel concevant la formation exclusivement sous l'angle d'une présentation dogmatique du droit national.

\section{Obstacles culturels}

On touche ici, au-delà des objections d'ordre pédagogique, à des raisons plus fondamentales, qui relèvent en quelque sorte du culturel, qui s'opposeraient à un tel renversement de perspective dans la formation des juristes. Ces raisons sont particulièrement prégnantes dans les pays qui sont marqués par une forte tradition juridique et où une longue et riche activité de systématisation doctrinale a abouti, à partir des textes et/ou de la jurisprudence, à la construction de systèmes élaborés et plus ou moins fermés vers l'extérieur. Je m'arrêterai ici à l'exemple du droit civil français parce que c'est celui que je connais un peu et parce que c'est en-

28. Sur ces difficultés, qui expliquent en partie que l'École de droit de Sciences Po Paris ait renoncé à établir dès la première année un programme de formation transnationalisé, voir C. JAMIN, préc., note 16, 239240. 
core un modèle de référence au Québec. Ce droit trouve ses racines dans un code particulièrement prestigieux, lui-même conçu, non comme une simple collection de textes, mais comme un système; mais, au moins dans les parties qui n'ont pas été modifiées depuis 1804 (le droit des biens et, surtout, le droit des obligations), il est aussi le produit d'une intense activité jurisprudentielle, qui, sur de nombreux points, a complètement transformé le sens de textes devenus obsolètes, et d'un considérable et incessant travail doctrinal qui cherche à introduire de la cohérence dans le fouillis des solutions à travers la construction d'un certain nombre de "théories " 29 . En France (mais sans doute aussi en Allemagne), c'est principalement à travers ce travail de systématisation des solutions qu'est pensé le rôle des professeurs de droit, tant dans leurs productions de recherche que dans leur activité d'enseignement. Dans son ouvrage déjà plusieurs fois cité, Christophe Jamin montre comment ce " modèle doctrinal " s'est fixé à la fin du XIXe siècle, à l'issue d'une grande crise où a été repensé le rôle des juristes dans l'interprétation des codes. Il montre aussi que ce modèle, plus ou moins abâtardi, n'a jamais été vraiment remis en cause, et qu'il bloque très largement les évolutions dans le domaine de l'enseignement du droit. Dans un tel modèle doctrinal, en effet, pour l'immense majorité des professeurs français, enseigner le droit civil, c'est former les étudiants au "système ", en en faisant précisément ressortir, au-delà des solutions de détail, la logique et la cohérence d'ensemble (logique et cohérence qu'ils contribuent largement à introduire eux-mêmes) ${ }^{30}$. À cela, qui fait la gloire, et, d'une certaine manière, comme l'a souligné l'un des promoteurs de l'enseignement transsystémique lui-même, la "valeur esthétique " du droit français ${ }^{31}$, la grande majorité des universitaires français ne sont sans doute pas prêts à renoncer. La

29. Comp. sur la rareté de ces "théories" en droit civil québécois : Mathieu DevinAt et Edith Guilhermont, "Enquête sur les théories juridiques en droit civil québécois ", (2010) 44-2 R.J.T. 11.

30. C. JAMIN, préc., note 16,42 s. et 151-163.

31. Nicolas KASIRER (alors doyen de la Faculté de droit de McGill), lors d'un débat radiophonique avec Horatia MUIR WATT dans l'émission animée par Antoine GARAPON (Le bien commun) en décembre 2007 (accessible sur le site Web de McGill: http://francais.mcgill.ca/crdpcq/transsystemic/ multimedia/). 
(2013) 43 R.D.U.S.

Quelle place

pour le droit national

109

dans l'enseignement du droit en Europe?

même observation pourrait sans doute être faite pour la plupart des " grands " pays européens, au moins ceux qui connaissent un système civiliste. S'interrogeant dans une revue allemande sur la possibilité de transposer en Europe un enseignement dénationalisé, Armand de Mestral et Helge Dedek observent ainsi que

in Europe, the "paradigm" of legal scholarship never completely shifted away from that of 19th century "legal science". This "paradigm" implies that what makes a great scholar is the mastery of law as a "system", and a command of the body of doctrine that surrounds the primary "sources of law". But the law-as-science paradigm also implies that judges and lawyers should employ the "scientific" approach in their work as well, and that students, too, should be brought up in its spirit. Mindset gravitates naturally towards the law that is actually in force, and, consequently, to a strong focus on jurisdictional boundaries. This is not conducive to a way of thinking about law as transcending the nation state ${ }^{32}$.

Nous sommes d'autant moins prêts à renoncer à ce modèle qu'une formation juridique "dénationalisée " ne peut pas rester sans influence sur le système juridique lui-même. L'enseignement du droit, en effet, n'est pas seulement la transmission aux futurs juristes des règles, des catégories, des techniques propres au système dans le cadre duquel ils exerceront leur activité. L'enseignement participe aussi, d'une certaine manière, à la production de ces règles, de ces catégories, de ces techniques, et donc à l'évolution - ou à la non-évolution - du droit enseigné, à la fois par l'influence des professeurs sur ceux qu'ils forment, par les orientations qu'ils leur donnent et qui peuvent contribuer à déterminer la manière dont ces futurs juristes, dans leur activité professionnelle, feront évoluer les interprétations et les pratiques, et aussi en raison du lien très étroit qui existe, au moins dans les pays de tradition civiliste, et spécialement en France, entre l'enseignement et l'activité doctrinale. À cet égard, enseigner un

32. A. De Mestral et H. DedeK, préc., note 7, 893-894. 
Quelle place

pour le droit national

(2013) 43 R.D.U.S.

dans l'enseignement du droit en Europe?

droit globalisé33, c'est d'abord participer à la promotion de règles juridiques transnationales dans lesquelles on ira volontiers chercher les solutions les plus appropriées, marquées du sceau de la modernité face aux solutions nationales affectées d'une certaine obsolescence : on peut penser par exemple que les étudiants de droit des contrats ainsi formés se tourneront sans doute davantage, dans leurs futures pratiques, contentieuses et non contentieuses, vers les Principes d'Unidroit, la Convention de Vienne sur la vente internationale, ou vers le droit commun européen de la vente actuellement en gestation ${ }^{34}$, au détriment de leur droit national dont le champ d'application pourrait ainsi se trouver de plus en plus réduit. Tout ce qui contribue à la promotion de ces droits transnationaux peut donc être (mal) vécu, dans les pays à forte tradition juridique, comme concourant à une certaine marginalisation du droit national: il suffit de songer aux réticences, voire à l'hostilité que suscitent dans la communauté doctrinale française, mais aussi dans d'autres doctrines de pays européens, les projets d'harmonisation européenne du droit des contrats et plus encore de la responsabilité délictuelle. Mais ensuite, de manière plus insidieuse, un enseignement dénationalisé peut avoir une incidence sur les structures mêmes du système national, qu'il

33. L'expression de droit global (global law) ou transnational est évidemment pleine d'ambiguïté. Il ne s'agit pas de désigner un système de droit complet et articulé à l'échelle mondiale, qui concurrencerait les droits nationaux, et qui pourrait à terme s'y substituer (une sorte de "droit commun mondial ") dont rêvaient les comparatistes au début du XXe siècle. Il s'agit plutôt d'une idée qui désigne à la fois l'internationalisation croissante des relations juridiques (d'où résulte une interpénétration constante des systèmes juridiques nationaux), la tendance au rapprochement des solutions de ces droits nationaux, et le développement de règles internationales unifiées d'origine publique (conventions internationales) ou privées (lex mercatoria).

34. Proposition de règlement du Parlement européen et du Conseil relatif à un droit commun européen de la vente (2011)1165 du 11 octobre 2011. Cette proposition vise à mettre en place un instrument optionnel, qui constituerait, dans chacun des États membres, un second régime pouvant être choisi par les parties (consommateurs et petites ou moyennes entreprises) à la place du droit national. Le texte ne concerne que la vente et les contrats de services liés, mais il contient de nombreuses dispositions relevant du droit commun des contrats, et pourrait bien constituer l'amorce d'une réglementation uniforme beaucoup plus générale. 
(2013) 43 R.D.U.S.

Quelle place

pour le droit national

dans l'enseignement du droit en Europe?

peut en quelque sorte contribuer à " corrompre " : non seulement parce qu'il est de nature à favoriser la pénétration dans ce système de multiples concepts venus d'ailleurs (l'enseignement du droit étant un des vecteurs de ce que $R$. A. Macdonald décrit comme la "contamination virale " d'un système ${ }^{35}$ ), mais aussi parce que, en privilégiant, dans une approche fonctionnelle, les différentes réponses possibles aux problèmes sociaux, on cesse de mettre au premier plan, dans la présentation qu'on en donne, la logique d'ensemble du système. Si on considère que, au-delà des solutions positives qu'il relie, le système n'a pas d'existence propre, qu'il n'est qu'un produit de l'activité de systématisation opérée par la doctrine, en liaison directe avec l'enseignement qui en est donné, on peut soutenir qu'une formation des juristes détachée d'un système aboutirait inéluctablement à saper les systèmes eux-mêmes. On peut dès lors comprendre la difficulté qu'il y aurait à implanter de manière généralisée des enseignements de ce type dans les pays porteurs d'une forte tradition juridique nationale. Cela, cependant, ne devrait pas conduire à remettre en cause, dans des cadres particuliers, des expérimentations de telles formations.

\section{Des expérimentations légitimes dans des contextes par- ticuliers}

Comme j'ai essayé de le montrer précédemment, des formations juridiques transnationalisées répondent d'ores et déjà à certains besoins en Europe, et elles pourraient présenter un intérêt même pour préparer des juristes ayant une activité principalement nationale face aux faiblesses des formations dogmatiques traditionnelles. Cependant, les nombreux obstacles à la mise en place de telles formations ne permettent pour l'instant de les envisager qu'à titre d'expériences limitées. Je voudrais ici, à partir de notre projet actuel dans le cadre luxembourgeois, proposer quelques réflexions sur les conditions dans lesquelles une telle

35. Roderick A. MACDONALD, "Three Metaphors of Norm Migration in International Context ", (2008-2009) 34 Brook. J. Int'l L. 603. 
expérimentation est envisageable (A) et sur les modalités qu'elle peut revêtir (B).

\section{A. Les conditions d'une expérimentation}

De mon point de vue, je l'ai dit, au-delà des difficultés pédagogiques qu'elles soulèvent, les obstacles au développement de formations transnationalisées en Europe sont principalement d'ordre culturel. Dès lors, la mise en place de telles formations apparaîtra plus facile dans des pays européens où la tradition juridique nationale est moins forte qu'elle n'est dans un pays comme la France ou l'Allemagne, soit que le droit national y soit le produit de l'importation plus ou moins volontaire d'un modèle étranger, soit qu'il soit en pleine reconstruction après les vicissitudes de l'histoire. Ces pays sont nécessairement plus ouverts vers les systèmes étrangers et vers le droit transnational, et sont sans doute plus réceptifs à l'idée de former des "juristes du monde ", qui ne seraient pas, d'abord, des spécialistes de droit national. Il serait intéressant d'observer à cet égard la manière dont se développe actuellement le droit, et l'enseignement du droit, dans les pays de l'ancien "bloc de l'Est ", surtout dans ceux où il n'existait pas, avant la période communiste, de tradition juridique assez solide pour résister à la tentative de socialisation du droit et où il a fallu, après la chute de l'empire soviétique, construire pratiquement à partir de rien un nouvel ordre juridique. L'exemple de l'Estonie, où la jurisprudence nationale fait officiellement place aux solutions d'autres systèmes juridiques, me semble particulièrement représentatif à cet égard ${ }^{36}$. En Europe occidentale, il est significatif que les deux seuls véritables exemples d'une formation juridique

dénationalisée au stade du bachelor, l'European Law School de Maastricht et le très récent bachelor "Global law " de Tilburg, se soient développés dans un pays comme les Pays-Bas, certes profondément marqué par la tradition française, mais où le nouveau Code civil de 1992 a marqué la volonté de reconstruire le système en mêlant aux éléments français du premier code les influences conjuguées du common law et du modèle germanique - un code

36. Voir à cet égard la contribution d'Irène Kull dans ce volume. 
(2013) 43 R.D.U.S.

Quelle place

pour le droit national

dans l'enseignement du droit en Europe?

d'une certaine manière " transnationalisé " qui permet de regarder la tradition historique avec plus de distance qu'en France ou en Allemagne. Et je me dois bien sûr d'évoquer ici l'exemple du Luxembourg où le projet de réforme du bachelor actuellement à l'étude est directement relié au contexte juridique très particulier de ce pays ${ }^{37}$.

Lorsqu'on enseigne à Luxembourg, on prend vite conscience que le droit national n'a pas nécessairement à occuper dans la formation des étudiants la même place que celle qui semble naturelle dans le cadre d'une faculté de droit française (ou allemande, ou anglaise). C'est que le droit national d'un petit pays, à peine grand comme un département français et peuplé d'un peu plus de 500000 habitants, ne peut pas être mis sur le même plan que les systèmes juridiques des "grands " pays voisins. $\mathrm{Si}$, dans certains secteurs (le droit des sociétés, le droit bancaire et financier) le Luxembourg a développé une législation spécifique (qui n'est pas pour rien dans l'attrait que le pays exerce auprès des investisseurs étrangers), il est en revanche resté très près du modèle français sur les matières de base du droit privé. Le droit civil, que j'y enseigne, y est fondé sur les mêmes bases textuelles que le droit français, puisque, comme la Belgique, le Luxembourg a conservé lors de son indépendance au XIX ${ }^{\mathrm{e}}$ siècle le Code civil français et que, au moins dans les matières patrimoniales (biens, obligations), les articles du Code sont restés très largement les mêmes dans les trois pays. Mais bien sûr, en raison du faible nombre d'habitants, le contentieux est quantitativement limité, et ces textes n'ont pas donné lieu aux mêmes développements jurisprudentiels qu'en France ou même que dans la Belgique voisine. Et, là encore à la différence de la France ou de la Belgique, ces textes n'ont jamais été éclairés, travaillés, systématisés par une doctrine proprement luxembourgeoise, ne serait-ce que parce que, jusqu'à une période récente, il n'existait pas d'université (donc pas de corps acadé-

37. Pour une présentation des études juridiques actuelles au Luxembourg on pourra consulter : Elise POILLOT, "Le particularisme de l'enseignement du droit au Luxembourg ", dans M. FlORES-LONJOU, C. LARONDE-ClÉRAC et A. DE LUGET (dir.), Quelle pédagogie pour l'étudiant juriste : Expérimentations, modélisations, circulation, Bruylant, 2012, 195-204. 
Quelle place

pour le droit national

(2013) 43 R.D.U.S.

dans l'enseignement du droit en Europe?

mique savant) au Luxembourg. Il en résulte que, sur de très nombreuses questions, celui qui enseigne serait bien en peine de dire ce qu'est la réponse propre du droit luxembourgeois. Il est alors amené, très souvent, à aller chercher des sources d'inspiration ailleurs, dans la doctrine et dans la jurisprudence françaises ou belges, comme le font du reste, de manière en quelque sorte " naturelle ", les juges luxembourgeois eux-mêmes formés jusque-là en France ou en Belgique. Cela donne à l'enseignement du droit à Luxembourg un caractère nécessairement, et d'emblée, comparatiste. Bien évidemment, pour les raisons précédemment évoquées, ce comparatisme "natif " reste très éloigné du transsystémisme, dans la mesure où il se meut principalement, pour l'instant du moins, à l'intérieur du cadre franco-belgo-luxembourgeois, qu'on peut considérer comme formant en lui-même un système au-delà des particularités nationales. Cependant, cette dépendance par rapport au modèle français n'a rien d'inéluctable. Dès lors que les réponses du droit luxembourgeois n'ont pas nécessairement à être les mêmes que celles du droit français, le professeur qui enseigne à Luxembourg a plus de liberté que celui qui, devant un auditoire français, se sent (à tort ou à raison) contraint de construire son enseignement à partir de la masse immense de la jurisprudence et de la doctrine françaises. À Luxembourg, on sera davantage porté, lorsqu'on traite de telle ou telle question de droit des contrats, à aller aussi chercher les réponses possibles dans le droit allemand, dans les Principes d'Unidroit, dans les différents projets doctrinaux européens ou dans la très récente proposition de règlement sur le droit commun européen de la vente... Même si elles ne sont pas aussi prégnantes, aussi naturelles que le modèle français, ces références n'ont évidemment rien d'incongru dans l'université d'une ville qui n'est pas seulement la capitale d'un petit État, mais qui est aussi le siège de la Cour de justice de l'Union européenne, en même temps qu'une place bancaire internationale.

Si le contexte juridique particulier d'un pays comme le Luxembourg, plus ouvert vers l'extérieur que celui des "grands pays " voisins, semble offrir un terrain favorable à une expérience de transnationalisation, il faut aussi tenir compte du contexte universitaire qui doit, lui aussi, présenter certaines caractéris- 
(2013) 43 R.D.U.S.

Quelle place

pour le droit national

dans l'enseignement du droit en Europe?

tiques pour rendre possible une telle expérience. Il convient à la fois de considérer le public à qui on s'adresse et la composition de l'équipe enseignante. Sur les deux plans, l'exemple luxembourgeois peut servir de point de départ utile à la réflexion, par comparaison avec ce que l'on peut observer dans les autres universités européennes, et spécialement par rapport à la situation française précédemment évoquée. Au Luxembourg, où près de la moitié des habitants sont des résidents étrangers, les étudiants que nous formons ont, d'ores et déjà, un profil très différent de celui que l'on rencontre dans les facultés de droit européennes de taille comparable. N'étant pas tous luxembourgeois, ils n'exerceront pas nécessairement leur activité sur place et ils iront du reste souvent compléter leur formation ailleurs. Même s'ils n'ont pas tous vocation à devenir des "global lawyers ", on peut supposer que tous ne viennent pas chercher à l'Université du Luxembourg une formation axée sur le seul droit national luxembourgeois, dont le champ d'application est nécessairement restreint. Un autre élément favorable est que, contrairement à ce qu'on observe dans les pays voisins, et spécialement en France, ces étudiants sont tous au minimum bilingues (français/anglais), et souvent trilingues (allemand) ${ }^{38}$, ce qui faciliterait évidemment l'accès à des enseignements où on jonglerait constamment avec des droits de pays différents ${ }^{39}$. Par ailleurs, pour l'instant, et alors même que l'Université ne pratique aucune sélection à l'entrée, les effectifs sont encore relativement peu nombreux, ce qui rend possible le développement de méthodes d'enseignement interactives indispen-

38. Le système d'enseignement primaire et secondaire au Luxembourg aboutit normalement à ce que les étudiants maîtrisent (plus ou moins bien) quatre langues : outre le luxembourgeois (langue nationale non utilisée sur le plan du droit), le français (langue officielle des lois et des décisions de justice), l'allemand (troisième langue officielle), et l'anglais (langue étrangère obligatoire).

39. Actuellement, les enseignements du bachelor en droit à l'Université du Luxembourg sont délivrés partie en français, partie en anglais. Autre différence notable avec les universités des pays voisins, où les cours sont délivrés presque exclusivement dans la langue nationale (français ou allemand). La pratique des cours en anglais semble cependant se répandre dans divers pays européens, notamment dans les pays d'Europe centrale qui peuvent ainsi attirer dans leurs universités certains étudiants étrangers. 
sables dans le cadre d'une formation transnationalisée ${ }^{40}$. Les membres de l'équipe pédagogique sont cependant conscients que certaines évolutions sont nécessaires pour capter un public plus réceptif à des enseignements transnationalisés. Au minimum, il paraît nécessaire d'introduire, sinon une véritable procédure de sélection, du moins un contrôle de motivation permettant d'éclairer à l'entrée les étudiants sur le contenu particulier de la formation dispensée, sur les difficultés qu'elle peut présenter, sur l'implication qu'elle requiert de la part de ceux qui la suivent ${ }^{41}$. Le but est à la fois de décourager un public local qui ne s'inscrit à Luxembourg que par commodité, mais aussi bien sûr d'attirer davantage d'étudiants extérieurs qui choisiraient de suivre un cursus original, complètement différent de celui qu'ils pourraient suivre dans les universités voisines des autres pays (France, Belgique, Allemagne) ${ }^{42}$.

Si on regarde maintenant du côté des enseignants, on note également d'importantes différences avec les universités des pays voisins. Dès sa création, l'Université du Luxembourg - et spécialement la Faculté de droit - a mis l'accent sur le caractère international du recrutement de ses enseignants : parmi les professeurs de droit de Luxembourg, certains ont été formés en France, d'autres en Allemagne, en Angleterre, en Italie, en Suisse, l'un en Afrique du Sud... et beaucoup, du moins dans la jeune génération,

40. Voir infra II.B.

41. Le site Web de 1'European Law School insiste avec raison sur cette exigence : "Although we do our very best to inform our prospective bachelor's students through brochures, open days and websites, we are unable to prevent some students starting our programmes with the wrong expectations. It might be that they misinterpret the information, or maybe their study choice was not well thought through. During their studies, some students find that their expectations do not match the reality of the programme and may subsequently decide to quit. This is a pity because they waste time and money on account of having made the wrong study choice. It is also problematic for the faculty, because poorly motivated students can negatively influence the performance of a tutorial group. Furthermore, reducing the drop-out rate generally is a key objective ".

42. C'est sans doute sur ce point que le projet actuellement à l'étude pourrait se heurter aux difficultés les plus grandes et rencontrer le plus de résistances. 
(2013) 43 R.D.U.S.

Quelle place

pour le droit national

dans l'enseignement du droit en Europe?

ont suivi pour leur propre formation un cursus international et ont déjà, avant de venir à Luxembourg, enseigné dans plusieurs pays. Il est clair qu'une telle internationalité de l'équipe, assez rare dans le monde universitaire européen, est de nature à favoriser la mise en place d'enseignements qui ne sont pas exclusivement axés sur le droit national. Certes, pour l'instant, il existe encore un certain cloisonnement en fonction des matières qui pourrait constituer un frein à une véritable transnationalisation des enseignements : par exemple, tous les professeurs de droit civil sont actuellement des professeurs français, qui pourraient être tentés de reproduire, dans leur enseignement, les concepts, les méthodes de raisonnement et d'argumentation qu'ils pratiquaient antérieurement. Il faut cependant observer que tous ces collègues, parce qu'ils ont choisi de venir travailler dans cette jeune université à vocation européenne et internationale, sont, sans renier leur formation d'origine, plus ouverts que la plupart des collègues restés dans leur milieu d'origine sur de nouveaux modèles de pensée et de formation ${ }^{43}$, et on peut penser qu'on passera assez vite d'un rassemblement hétérogène de professeurs d'origines diverses à une véritable communauté universitaire.

\section{B. Les modalités d'une expérimentation}

Même dans un contexte a priori favorable, la mise en place d'un cursus transnational ne s'improvise pas, elle suppose une réorganisation complète des programmes d'enseignement, sans doute aussi une évolution profonde des méthodes pédagogiques. Sur le premier point, la question essentielle est de savoir comment il faut articuler la formation transnationale avec les enseignements portant sur le droit national ${ }^{44}$, lesquels restent pour moi de

43. Comp. pour le cas de Sciences Po Paris : C. JAmin, préc., note 16, 250254.

44. Ce n'est pas, évidemment, la seule question. Je laisse ici de côté, pour ne pas alourdir ce texte, d'autres questions également importantes qui tiennent à la place que devraient occuper, dans un tel cursus, l'histoire du droit (et quelle histoire du droit?), la théorie et/ou la philosophie du droit, ainsi qu'au rôle qu'il faudrait y donner à l'interdisciplinarité (sociologie du droit, law and economics, etc.). Sur ce dernier aspect voir les critiques faites au programme de McGill par Harry ARTHURS, "Madly off in 
toute façon nécessaires. Même dans le cadre d'expériences limitées, il ne m'apparaît pas envisageable de former des juristes qui seraient seulement des juristes du monde, en les privant de toute attache identitaire. D'une part, sur le plan des débouchés professionnels, beaucoup des juristes que nous formons continueront d'exercer leur activité à l'intérieur d'un système juridique national, et même ceux qui feront principalement une activité de juriste international devront généralement, pour y accéder, passer sous les fourches caudines d'un examen professionnel dans le cadre duquel, pour de longues années encore, la connaissance du droit national sera privilégiée. D'autre part, plus fondamentalement, on peut penser que les enseignements transnationaux ne suffisent pas, à eux seuls, à permettre aux étudiants de développer les compétences suffisantes pour devenir de vrais juristes, pour "penser comme des juristes" 45 . Ces enseignements permettent sans doute aux étudiants d'acquérir une ouverture leur permettant de "naviguer " plus facilement dans un monde globalisé et dans une Europe en voie d'harmonisation, ils peuvent, aussi, leur donner davantage d'esprit critique et d'imagination créatrice par rapport à leur propre système que ne le permet la formation traditionnelle. Mais on peut douter que ces enseignements soient aptes à développer la maîtrise technique du raisonnement, de l'interprétation, de la résolution de cas, de la combinaison de règles et de concepts qui est au cœur du travail d'un juriste. Cela, qui relève de l'application du droit, de sa mise en œuvre, ne peut se faire, selon moi, qu'en se plaçant à l'intérieur d'un système déterminé de droit national - tant du moins que les systèmes nationaux n'auront pas été complètement remplacés par des règles internationales harmonisées (ce qui, on s'en doute, n'est pas pour demain!).

one Direction - McGill's New Integrated, Polyjural, Transsystemic Law Programme ", (2005) 50 R.D. McGill 707; adde Vincent FORRAY, "Enseigner le droit complexe, redéfinir le droit en compétence. Sur une analyse américaine du programme transsystémique de McGill ", (2010) Jurisprudence - Revue critique, 267.

45. Allusion à l'ouvrage classique de Kenneth J. VANDEVELDE, Thinking Like a Lawyer: An Introduction to Legal Reasoning, Westview Press, 2e éd., 2010. 
(2013) 43 R.D.U.S. $\quad \begin{gathered}\text { Quelle place } \\ \text { pour le droit national } \\ \text { dans l'enseignement du droit en Europe? }\end{gathered}$

Cette combinaison entre les enseignements transnationaux et ceux qui portent sur le système national peut être assurée de plusieurs manières. D'une part, il n'est évidemment pas nécessaire que, dans un cursus transnational, toutes les matières fassent l'objet d'un enseignement de type transsystémique : celui-ci ne s'impose que dans certaines matières, qui sont à la fois les plus fondamentales pour la formation des étudiants et celles où les problématiques sont suffisamment universelles pour qu'on puisse espérer aboutir à une modélisation pertinente des solutions. Ainsi, à Luxembourg, dans le cadre de notre réflexion sur le projet de réforme du bachelor, nous avons estimé que le droit constitutionnel, le droit des contrats, le droit de la responsabilité, le droit des sociétés, le droit pénal se prêtaient particulièrement bien à l'opération, laquelle semblait, dans le contexte local, beaucoup plus difficile à mettre en ouvre pour le cours de droit administratif ou pour le cours de droit du travail - non pas parce que ces cours seraient, en eux-mêmes, inaptes à être transnationalisés, mais en raison de la composition de l'équipe pédagogique. D'autre part, même dans les matières faisant l'objet d'une transnationalisation, il faut songer à doubler les enseignements transnationaux d'enseignements portant sur le droit national. La formule la plus simple consiste à proposer (ou à imposer) aux étudiants, à côté des cours transnationaux, des cours de droit national, soit en parallèle, soit, d'une manière plus conforme à l'esprit de ce type de formation, dans un second temps du cursus. C'est la formule choisie à McGill, où, après une première année où dominent les enseignements transystémiques, les étudiants se voient proposer dans les mêmes matières (contrats, responsabilité) des cours de common law et de droit civil québécois qu'ils choisissent en option. De même, à Maastricht, après une première année entièrement transnationalisée, on remarque l'introduction en deuxième année de la formation de cours d'introduction au droit néerlandais ${ }^{46}$. À Luxembourg, nous réfléchissons actuellement à une autre formule, qui consisterait à assurer la formation au droit national à

46. Voir le programme des cours sur http://www.maastrichtuniversity.nl/ web/Faculties/FL/TargetGroup/ProspectiveStudents/BachelorsProgram mes/EuropeanLawSchoolEnglishLanguageTrack/CourseDescriptions. htm. 
travers les travaux dirigés, où les étudiants apprendraient, sur des questions de détail, à rechercher, à analyser et à combiner des textes et de la jurisprudence et à les appliquer à des situations de fait. Contrairement à la pratique actuelle, ces exercices d'application du droit, menés à l'intérieur du système national, seraient a priori déconnectés du cours dont le contenu serait transnational - même si, bien sûr, ils pourraient être aussi l'occasion de montrer aux étudiants l'étroite imbrication des solutions nationales et des règles européennes et internationales. On pourrait cependant concevoir que ces travaux dirigés soient précédés d'une courte présentation, en quelques heures, de la logique d'ensemble de la matière - présentation qui servirait en quelque sorte de guide d'orientation aux étudiants et qui leur permettrait de prendre conscience de l'articulation des différentes solutions au sein d'un système.

On voit par là que la réflexion sur le contenu des enseignements, dans un cursus transnational, ne peut pas faire abstraction de la question des méthodes mises en œuvre pour enseigner. Dans la plupart des pays d'Europe continentale, la formule du cours magistral, éventuellement complété par des travaux dirigés, est très largement privilégiée. Si elle est évidemment commandée par certaines conditions matérielles de l'enseignement (auditoires pléthoriques, locaux insuffisants, difficultés financières des universités...) qui rendent difficile la généralisation des enseignements interactifs par petits groupes, cette formule est aussi, je crois, profondément liée aux caractéristiques du système juridique des pays de droit civil, en même temps qu'elle paraît être un moyen de le pérenniser face à la concurrence des droits de common law. Système au sens plein du terme, dans lequel le droit est conçu comme un ensemble de règles articulées les unes avec les autres, et formant un ordonnancement supposé cohérent. Pour présenter un tel système, pour en faire comprendre, précisément, la cohérence, le cours magistral, où le professeur expose de manière unilatérale l'état du droit sous la forme d'un discours continu et organisé, paraît de prime abord la formule d'enseignement la plus adaptée. Paradoxalement, l'adjonction au cours magistral de travaux dirigés, qui se rencontre dans de nombreux pays, paraît 
(2013) 43 R.D.U.S.

Quelle place

pour le droit national

dans l'enseignement du droit en Europe?

de nature à renforcer encore le côté dogmatique et systématique de l'enseignement, dans la mesure où ces travaux dirigés sont conçus comme des enseignements complémentaires, venant après le cours, et destinés à appliquer les connaissances délivrées en cours, les exercices proposés aux étudiants apparaissant comme autant de confirmations du système préalablement exposé par le professeur. Il est clair qu'une telle organisation apparaît peu adaptée à un enseignement transnational, dont l'objet n'est pas de présenter aux étudiants les solutions positives et la manière dont elles s'articulent (ou sont censées s'articuler) entre elles, mais de les faire réfléchir, de manière comparative, sur les différentes manières de résoudre un certain nombre de problèmes. Dans cette optique, il paraît indispensable de renoncer à la formule traditionnelle des cours magistraux et de les remplacer par des enseignements interactifs. Cela suppose la division des effectifs étudiants en groupes restreints, la préparation préalable des sujets enseignés par les étudiants et, en conséquence, une diminution sensible des heures d'enseignement pour laisser du temps à ce travail de préparation... Autant de choses qui sont assez loin de ce qui est actuellement pratiqué dans la plupart des universités européennes, et qui, même dans un cadre a priori favorable comme celui de l'Université du Luxembourg, peuvent susciter des difficultés.

C'est dire que, même dans le cadre d'expériences limitées, la mise en place d'une formation juridique transnationale - ou comportant une certaine dose de transnationalisation - est un vaste chantier, qui ne doit être conduit qu'avec une grande prudence et de manière progressive. Les risques et les difficultés de telles expériences ne doivent cependant pas dissuader de s'y lancer lorsque le contexte apparaît favorable. Au-delà des apports que ces formations peuvent présenter pour les publics forcément restreints qu'elles concerneront dans un premier temps, elles peuvent constituer un point d'ancrage pour réfléchir aux faiblesses de la formation des juristes dans le monde contemporain et pour contribuer aux indispensables évolutions futures. 
